\title{
Re-Sequencing and Transcriptomic Analysis Reveal Differences in Nitrite Reductase in Jujube Fruit (Ziziphus Jujube)
}

$\mathrm{Na} \mathrm{Li}$

Shanxi Agricultural University

Yuqin Song

Shanxi Agricultural University

Jie Li

Shanxi Agricultural University

Ruijie Hao

Shanxi Agricultural University

Xinxin Feng

Shanxi Agricultural University

Liulin Li ( $\square$ tgliulin@163.com )

Shanxi Agricultural University https://orcid.org/0000-0003-0554-2311

\section{Research}

Keywords: Ziziphus jujube, transcriptome, genome re-sequence, indel, nitrite reductase

Posted Date: April 16th, 2021

DOI: https://doi.org/10.21203/rs.3.rs-419637/v1

License: (a) This work is licensed under a Creative Commons Attribution 4.0 International License. Read Full License

Version of Record: A version of this preprint was published at Plant Methods on July 12th, 2021. See the published version at https://doi.org/10.1186/s13007-021-00776-9. 


\section{Abstract}

Background

Jujube is one of the characteristic fruit tree species in China. 'Linhuang No. 1', a cracking-resistant cultivar, and 'Muzao', a crackingsusceptible cultivar, were selected as materials by previous study. Whole-genome re-sequencing and transcriptome of 'Linhuang No. 1' and 'Muzao' allow the screening out of differently expressed genes with different gene structures between them. It could be helpful in explaining divergence/similarity between the two cultivars.

Results

There are 664,129 mutation sites between 'Linhuang No. 1 ' and 'Muzao' by re-sequencing. To determine the genetic relationship of 'Linhuang 1', 'Muzao' and reference genome 'Dongzao', the characteristic mutation sites were analyzed by principal component analysis. The genetic relationship between 'Linhuang No. 1' and 'Muzao' was closer than that with 'Dongzao'. 19 differentially expressed genes were screened by combining the transcriptomics with re-sequencing analysis. LOC107427052 (encoding nitrite reductase) was determined by KEGG enrichment analysis for further study. The large base insertion was not in the domain region of the LOC107427052 gene CDS region. As verified by the finding that the base insertion did not affect protein translation. LOC107427052 gene expression levels, the nitrite reductase activities and the nitrite content of 'Muzao' were significantly higher than those of 'Linhuang No. 1' at young fruit stage. There was no significant difference in the product ammonia of nitrite reductase between the two varieties.

Conclusions

Our study has laid a foundation for the analysis of genetic information and the comparative nitrite metabolism of 'Linhuang No. 1' and 'Muzao'.

\section{Introduction}

Jujube is one of the characteristic fruit tree species in China[1]. Jujube is valued as a traditional herbal medicine, and is cultivated on 2 million hectares in China alone, with an annual production of approximately 7.36 million tons in 2018

(https://data.stats.gov.cn/easyquery.htm?cn=E0103). Jujube cv. Muzao is one of the most widely cultivated jujube varieties in China, combining the characteristics of high yield and high quality[2]. Due to the wide cultivation area of the Muzao tree crop (Ziziphus jujube Mill.), there are many varieties and geographical types of jujube, varying in terms of fruit shape, single fruit weight, drying rate, nutrient composition and fruit characteristics[3]. 'Linhuang No. 1' is a dry-jujube cultivar selected from 'Muzao', which has fruit characteristics similar to those of 'Muzao'. Compared with 'Muzao', 'Linhuang No. 1' shows greater resistance to fruit cracking[4]. 'Linhuang No. 1' has become a valuable genotype for scientific research.

Whole-genome resequencing is the process of sequencing the genomes of different individuals of a species where the genome has already been sequenced[5]. Through sequence alignment, this methodology is able to identify large numbers of single nucleotide polymorphisms (SNPs), insertion/deletion (indel) variants, structural gene variants (SV), copy number variants (CNV) and other mutations in the wholegenome re-sequenced individuals. This information on genetic variation is an important way to understand the genetic background of a species and to study its evolution. In order to explore the wide phenotypic diversity of sweet cherry varieties with respect to important agronomic traits, such as flowering time and defense response to pathogens, Aliki et al. (2020) re-sequenced 21 cherry accessions and found that the majority of high-impact SNPs (e.g., addition of stop codon, frameshifts) were identified in genes involved in flowering time, dormancy and defense reactions against pathogens[6]. Yu et al. (2018) re-sequenced the genomes of 58 peach cultivars and closely related species to explore the origin and evolutionary history of peaches[7]. The results showed that peaches originated on the Qinghai-Tibet Plateau in Southwest China and had been subjected to frugivore-mediated selection to drive the evolution of fruit traits. The publication of the 'Dongzao' genome sequence provided valuable help for genomic and transcriptomic studies of other jujube cultivars[8]. The genome sequence of jujube cv. Dongzao represents a valuable resource not only for biological discovery and crop improvement but also for evolutionary and comparative genomic analysis. Huang et al. (2016) re-sequenced the genomes of 31 cultivated and wild jujubes to reveal the domestication process of jujube and screened key genes for metabolic effects associated with sweet or sour fruit[9].

In this article, we present analyses of whole-genome re-sequencing and transcriptome of 'Linhuang No. 1' and 'Muzao'. The sequence analysis focused on genomic regions associated with propitious variation, such as single nucleotide polymorphisms (SNPs) and insertions/deletions (InDels). The analysis focused on genes differentially expressed during the full-red period of fruit development. The combination of whole-genome re-sequencing and transcriptome analysis would allow the screening out of differently expressed genes with different gene structures between 'Linhuang No. 1' and 'Muzao', which could be helpful in explaining divergence/similarity of cracking resistance between the two cultivars. 
Results

\section{Discovery of SNPs and InDels in 'Linhuang No.1' and 'Muzao'}

A total of 110 million raw reads were generated for each of 'Linhuang No. 1' and 'Muzao'. After removal of low-quality reads, about 95.93\% ('Linhuang No. 1') and 94.74\% ('Muzao') of the reads were retained as clean data and used for further investigation. The Q20 percentage (proportion of nucleotides with a quality value greater than 20 in the reads) was greater than $95 \%$ and the Q30 percentage was greater than $89 \%$. The mean GC percentage of the clean reads was $33 \%$. The high-quality reads were further mapped onto the reference genome (Ziziphus jujube 1.1), using BWA software. Overall, almost $94 \%$ of these reads were uniquely mapped, and covered about $46.5 \%$ of the reference genome, with at least $30 \times$ coverage depth (Supplementary materials 1 ).

Additional file 1: Table S1. Summary of sequencing and mapping results

Compared with the $Z$. jujuba reference ('Dongzao') genome sequence, a total of 4,404,985 polymorphic sites including 3,623,127 SNPs and 781,858 indels were discovered in 'Linhuang No. 1', with a total of 4,401,491 polymorphic sites, including 3,626,419 SNPs and 775,072 indels, in 'Muzao'. From the comparison between 'Linhuang No. 1' and 'Muzao', 664,129 polymorphisms were detected. Table 1(A) summarizes the number of SNP and indel variants relative to the reference genome sequence and according to chromosome, whereas Table 1 (B) details the number of polymorphisms between 'Linhuang No. 1' and 'Muzao' by chromosome. The highest number of SNPs and indels between 'Linhuang No. 1' and the reference genome was detected for NC_029679.1 (Chromosome 1), whereas the lowest number of SNPs and indels was detected for NC_029690.1 (Chromosome 12). For all chromosomes, indel polymorphisms were less frequent than SNPs. From the comparison between 'Muzao' and the reference genome, the highest and lowest number of SNPs and indels were also detected on Chromosomes 1 and 12, respectively. From the comparison between 'Linhuang No. 1' and 'Muzao', the highest and lowest numbers of SNP and indel polymorphisms were also found on Chromosomes 1 and 12, respectively.

Table 1

Number of SNPs and indels, according to chromosome (Ch), detected for 'Linhuang No. 1' and 'Muzao', relative to the reference genome 'Dongzao' (A), and the comparison between them (B).

\begin{tabular}{|c|c|c|c|c|c|c|c|c|c|c|c|}
\hline \multirow[t]{2}{*}{ A) } & & & & & \multicolumn{7}{|l|}{ B) } \\
\hline & 'Linhuang & No.1' & 'Muzao' & & Linhuang & No.1' vs 'I & uzao' & & & & \\
\hline \multirow[t]{2}{*}{$\mathrm{Chr}$} & SNP & InDel & SNP & InDel & SNP & InDel & & & & & \\
\hline & & & & & & $\leq 10 \mathrm{bp}$ & $\begin{array}{l}11- \\
20 \mathrm{bp}\end{array}$ & $\begin{array}{l}21- \\
40 \mathrm{bp}\end{array}$ & $\begin{array}{l}41- \\
80 \mathrm{bp}\end{array}$ & $\begin{array}{l}81- \\
160 \mathrm{bp}\end{array}$ & $\geq_{161 b p}$ \\
\hline NC_029679.1 & 429504 & 89039 & 430221 & 88650 & 47822 & 12968 & 1437 & 930 & 780 & 310 & 43 \\
\hline NC_029680.1 & 246814 & 54159 & 246954 & 53764 & 30426 & 8489 & 877 & 567 & 466 & 218 & 30 \\
\hline NC_029681.1 & 245843 & 54834 & 245635 & 54282 & 29879 & 8761 & 921 & 553 & 495 & 174 & 26 \\
\hline NC_029682.1 & 228631 & 48207 & 229082 & 47855 & 32212 & 8774 & 899 & 574 & 446 & 199 & 31 \\
\hline NC_029683.1 & 343538 & 69172 & 344093 & 68807 & 45243 & 12055 & 1303 & 776 & 615 & 222 & 40 \\
\hline NC_029684.1 & 217230 & 47474 & 217519 & 46967 & 25026 & 7109 & 812 & 510 & 409 & 167 & 31 \\
\hline NC_029685.1 & 251890 & 52302 & 252798 & 52,148 & 37682 & 9423 & 991 & 659 & 510 & 185 & 39 \\
\hline NC_029686.1 & 182760 & 42746 & 182578 & 42,337 & 22962 & 6699 & 688 & 445 & 389 & 169 & 29 \\
\hline NC_029687.1 & 240326 & 55181 & 239869 & 54,513 & 29621 & 8750 & 814 & 540 & 461 & 171 & 36 \\
\hline NC_029688.1 & 195933 & 41950 & 196006 & 41,515 & 22887 & 6562 & 733 & 471 & 358 & 149 & 28 \\
\hline NC_029689.1 & 189397 & 43820 & 189007 & 43,509 & 24530 & 6804 & 776 & 468 & 322 & 129 & 22 \\
\hline NC_029690.1 & 155137 & 35962 & 155452 & 35,687 & 18755 & 5599 & 630 & 393 & 287 & 137 & 29 \\
\hline $\begin{array}{l}\text { unplaced } \\
\text { genomic } \\
\text { scaffold }\end{array}$ & 696124 & 147012 & 697205 & 145,038 & 127817 & 34303 & 3223 & 1936 & 1323 & 510 & 60 \\
\hline Total & 3623127 & 781858 & 3626419 & 775,072 & 494862 & 136296 & 14104 & 8822 & 6861 & 2740 & 444 \\
\hline
\end{tabular}


Figure 1 shows the relatedness among 'Linhuang No.1', 'Muzao' and 'Dongzao'. The distance between the points represents the degree of characteristic differences among the accessions. The first and the second principal components (PC1 and PC2) explained 58.22\% and $31.92 \%$ of the total variation, respectively. The cumulative contribution rate of the two principal components was $90.14 \%$. PC1 differentiated 'Linhuang No. 1' and 'Muzao' from 'Dongzao', whereas PC2 differentiated 'Linhuang No. 1', 'Muzao' and 'Dongzao'. From the comparison between 'Linhuang No. 1' and 'Muzao', 664,129 polymorphisms were detected.

SNP and indel density plots, obtained through the comparison of 'Linhuang No. 1' and 'Muzao' against the reference genome 'Dongzao', are displayed in Fig. 2. This figure also provides an integrated view of the polymorphisms detected across the entire genome between 'Linhuang No. 1' and 'Muzao' in relation to the reference genome. 'Linhuang No. 1' has a similar level of polymorphism to 'Muzao', when compared with the reference genome.

\section{Differential expression of different structural genes between 'Linhuang No. 1' and 'Muzao'}

In order to further explore the differential expression of variant genes between 'Linhuang No. 1' and 'Muzao', combined resequencing and transcriptome analysis (SRP307646) of 'Linhuang No. 1' and 'Muzao' was carried out. The results showed that there were 33 and 29 SNP and indel variant genes, respectively, differentially expressed in 'Linhuang No. 1' and 'Muzao'. A total of 947 genes in 'Linhuang No. 1' and 'Muzao' were mutated by SNP or indel, and 19 genes were differentially expressed between the two cultivars (Fig. 3.A).

We performed cluster analysis on the 19 DEGs (Fig. 3.B). We analyzed clustering of the log2 (Fragments per Kilobase Million, FPKM) of the gene expression. A color closer to red represents higher gene expression. Of the 19 DEGs, 12 genes were up-regulated in 'Muzao', including LOC107413306, LOC107427052, LOC107431206, LOC107432789, LOC107427373, LOC107404912, LOC107428467, LOC107426536, LOC107414473, LOC107434555, LOC107405940, and LOC107405347.

The 19 differentially expressed genes were further enriched by KEGG analysis (Fig. 3. C, Table 2). According to the standard of $p<0.05$, there were three enriched pathways in 'Linhuang No. 1' and 'Muzao', namely stilbenoid, diarylheptanoid and gingerol biosynthesis (Rich factor: 14.98), flavonoid biosynthesis (Rich factor: 8.85) and nitrogen metabolism (Rich factor: 19.48). Nitrogen metabolism (LOC107427052) was selected to further verify the transcriptomic data. 
Table 2

Enriched pathways of 19 differentially expressed genes

\begin{tabular}{|c|c|c|c|}
\hline & pathway ID & pathway & $p$-value \\
\hline 1 & ko00945 & Stilbenoid, diarylheptanoid and gingerol biosynthesis & 0.00697 \\
\hline 2 & ko00941 & Flavonoid biosynthesis & 0.01965 \\
\hline 3 & ko00910 & Nitrogen metabolism & 0.04939 \\
\hline 4 & ko00450 & Selenocompound metabolism & 0.06990 \\
\hline 5 & ko00940 & Phenylpropanoid biosynthesis & 0.07128 \\
\hline 6 & ko09106 & Metabolism of other amino acids & 0.10862 \\
\hline 7 & ko00270 & Cysteine and methionine metabolism & 0.13537 \\
\hline 8 & ko04626 & Plant-pathogen interaction & 0.17866 \\
\hline 9 & ko09159 & Environmental adaptation & 0.20606 \\
\hline 10 & ko09150 & Organismal Systems & 0.20606 \\
\hline 11 & ko09110 & Biosynthesis of other secondary metabolites & 0.26609 \\
\hline 12 & ko00460 & Cyanoamino acid metabolism & 0.26972 \\
\hline 13 & ko04144 & Endocytosis & 0.38498 \\
\hline 14 & ko09102 & Energy metabolism & 0.38498 \\
\hline 15 & ko04075 & Plant hormone signal transduction & 0.40467 \\
\hline 16 & ko09140 & Cellular Processes & 0.49486 \\
\hline 17 & ko09141 & Transport and catabolism & 0.49486 \\
\hline 18 & ko04131 & Membrane trafficking & 0.52736 \\
\hline 19 & ko09132 & Signal transduction & 0.56775 \\
\hline 20 & ko09105 & Amino acid metabolism & 0.56775 \\
\hline 21 & ko09100 & Metabolism & 0.63880 \\
\hline 22 & ko09130 & Environmental Information Processing & 0.67476 \\
\hline 23 & ko09182 & Protein families: genetic information processing & 0.97181 \\
\hline 24 & ko09180 & Brite Hierarchies & 0.99815 \\
\hline
\end{tabular}

\section{Levels of nitrite reductase (NiR) activity, nitrite and ammonia nitrogen concentration}

LOC107427052 encodes nitrite reductase. In the process of assimilating nitrate nitrogen in plants, nitrite reductase (NiR) is coupled with nitrate reductase (NR) to complete the inorganic assimilation of nitrate. Nitrite reductase can catalytically reduce nitrite to ammonia. Nitrite reductase plays a very important role in the growth and development of plants and is widespread in the leaves and roots of higher plants. Based on the re-sequencing of the genomes of 'Linhuang No. 1' and 'Muzao', it was found that indel events occurred in the LOC107427052 sequences of both 'Linhuang No. 1' and 'Muzao', compared with 'Dongzao' (Fig. 4). Some 106 bases were inserted at position 12517784 (in the coding sequence (CDS) region) on chromosome NC_029687.1 of 'Linhuang No. 1', while 104 bases were inserted at the same position of 'Muzao'. In order to confirm the results of re-sequencing, the LOC107427052 gene was cloned, using cDNA of 'Linhuang No. 1' and 'Muzao'. The protein sequences encoded by the LOC107427052 gene of 'Dongzao', 'Linhuang No. 1' and 'Muzao' were compared, and the similarity was $91.82 \%$ (Supplementary Fig. 1). Domain prediction was performed on the protein sequence, and it was found that 'Linhuang No. 1' and 'Muzao' base insertion did not occur in the domain region (Supplementary Fig. 2). Real-time PCR was used to verify the expression level of the LOC107427052 gene (Fig. 5). The results showed that the LOC107427052 gene expression levels of 'Muzao' were significantly higher than those of 'Linhuang No. 1' during fruit development, and that the expression levels of the LOC107427052 gene in 'Linhuang No. 1' and 'Muzao' increased gradually as the fruit developed. The expression level of the LOC107427052 gene was significantly higher during the coloring period and the full-red period than in the young fruit period. 
Additional file 2: Figure S1. Sequence alignment of 'Dongzao', 'Linhuang No. 1' and 'Muzao' LOC107427052 gene

Additional file 3: LOC107427052 gene protein sequence of 'Dongzao', 'Linhuang No. 1' and 'Muzao'

Additional file 4: Figure S2. Domain prediction of 'Dongzao', 'Linhuang No. 1' and 'Muzao' LOC107427052 gene

At the same time, the nitrite reductase activities of 'Linhuang No. 1' and 'Muzao' at different developmental stages were determined. The results showed that there was no significant difference in the nitrite reductase activities between 'Linhuang No. 1' and 'Muzao' at the later stage of fruit development; The nitrite reductase activities of 'Linhuang No. 1' and 'Muzao' were $0.75 \mathrm{U} / \mathrm{g}$ fresh weight (FW) and $0.93 \mathrm{U} / \mathrm{g}$ FW at the full-red stage, respectively. But the nitrite reductase activities of 'Muzao' was significantly higher than that of 'Linhuang No. 1' at young fruit stage. The nitrite content of 'Muzao' at the young fruit stage was significantly higher than that of 'Linhuang No. 1'. There was no significant difference in the product ammonia of nitrite reductase between the two varieties.

\section{Discussion}

The Chinese jujube tree was domesticated from the wild jujube (Z. jujuba Mill. var. spinosa Hu.)[10]. Cultivated jujube trees and wild jujube showed different characteristics, such as tree vs. shrub habit, sparsely thorned vs. heavily thorned, and large vs. small fruits, respectively, through artificial selection for important agronomic characters[8, 11, 12]. Most cultivated jujube varieties produce relatively fewer seeds due to self-incompatibility or cross-incompatibility. Jujube trees can only be bred through seed progeny selection, selection by bud mutation or molecular breeding[13]. These genetic methods resulted in the high heterozygosity, high repeat sequence density and low GC content of the jujube genome[13]. Therefore, sequencing of the jujube genome was very difficult. Liu et al. (2014) published the genome of the first cultivar of the genus Ziziphus, 'Dongzao' (437.65 Mbp)[8]. The release of this genome sequence provided a rich resource of genetic information for the breeding of improved jujube accessions, as well as for the molecular improvement of other plants and fruit trees of the family Rhamnaceae.

Huang et al. (2016) reported the genome sequence of another jujube variety, 'Junzao', and re-sequenced the genomes of 31 wild jujube and jujube accessions with different geographical distributions[9]. The research of Huang and colleagues revealed the genomic mechanism underlying improvement of fruit sweetness and acidity during domestication and identified four genes related to acidic metabolism pathways, encoding NADP-dependent malic enzyme, pyruvate kinase, isocitrate dehydrogenase and aconitate hydratase, which play key roles in organic acid metabolism. This research provides valuable genomic information and material for jujube breeding. Guo et al. (2020) reported the re-sequencing of 350 jujube accessions[13]. Through a genome-wide association study (GWAS) and selective sweep analysis, variation in the genes involved in the regulation of seven domestication traits were screened for, including fruit shape, kernel size and fruiting branch length. This study provided rich genomic resources for revealing the genetic basis of the domestication and evolution of jujube.

In the current study, the varieties 'Linhuang No. 1' (resistant to cracking) and 'Muzao' (susceptible to cracking) were used as the research materials, with 'Dongzao' used as the reference genome for resequencing analysis. It was found that 4,404,985 and 4,401,491 polymorphic sites were obtained in 'Linhuang No. 1' and 'Muzao', respectively, whereas 664,129 variant sites were found between 'Linhuang No. 1' and 'Muzao'. Principal component analysis on the characteristic variant sites between 'Linhuang No. 1', 'Muzao' and 'Dongzao' were performed and the results showed that both PC1 and PC2 could distinguish 'Linhuang No. 1' and 'Muzao' from 'Dongzao', indicating that 'Linhuang No. 1 ' and 'Muzao' were closer in relation to one another than to 'Dongzao'.

Mutation is a key element in species evolution. The widespread base substitution and insertion/deletion mutations are important driving forces for genome evolution[14, 15]. Insertion and deletion mutations are more likely to trigger species evolution than are base mutations. The greater the evolutionary distance between species, the more bases are inserted or deleted, and the greater the length of the insertions or deletions[16-18]. Insertions and deletions are the main reasons for the divergence of closely related species. The insertion and deletion of bases can cause DNA sequence changes and cause DNA fragment length polymorphisms. They can even change the structure of genes through insertions or deletions in the exons and introns of the original gene, leading to the generation of new genes in the genome[19]. It was found, through re-sequencing, that there were 781,858 and 775,072 base insertions or deletions in 'Linhuang No. 1' and 'Muzao', respectively. Compared with 'Linhuang No. 1', there were 169,267 base insertions and deletions in 'Muzao'. To a large extent, this led to the different characteristics between 'Linhuang 1'and 'Muzao'.

The transcriptome combines the genetic information of the genome with that of the proteome, with biological functions based on RNA, uses high-throughput sequencing technology (RNA-Seq) to sequence all cDNA libraries in tissues or cells and calculates the gene expression under different processing conditions (by counting the number of relevant reads). The jujube transcriptome has been widely used in research into response to heat stress, cold stress, salt stress and coloring of jujube fruits[20-22]. In the current study, 431 differentially expressed genes were identified by transcriptome analysis of 'Linhuang No. 1' and 'Muzao' pericarps. At the same time, the transcriptome and resequencing

Page 6/14 
analyses were combined to screen for differential expression of 'Linhuang No. 1' and 'Muzao' genes with respect to gene structural mutations. A total of 19 mutant genes were screened for differential expression. There were three significantly enriched pathways in 'Linhuang No. 1' and 'Muzao': stilbenoid, diarylheptanoid and gingerol biosynthesis (Rich factor: 14.98), flavonoid biosynthesis (Rich Factor: 8.85) and nitrogen metabolism (Rich factor: 19.48). The gene LOC107427052, encoding nitrite reductase, was screened for further study.

In the process of nitrate assimilation in plants, nitrite reductase (NiR) is coupled with nitrate reductase (NR) to complete the inorganic assimilation of nitrate. Nitrite reductase can catalytically reduce nitrate to ammonium[23]. In fact, the reduction reaction rate of nitrate must be strictly regulated. It is necessary to ensure that nitrite and ammonium will not be excessive to avoid plant poisoning, and the supply of ammonium must be ensured. In this process, NiR plays a role in connecting the past and the future. Sivasankar et al. found that the nitrate inducibility is a gene located between the upstream 230 and 180 positions, and the downstream 1 to 67 positions are very important for the minimum induction of nitrate[24]. Ozawa and Kawahigashi (2005) cloned the NiR gene of rice 'Konansou' and overexpressed the gene in a commercial rice variety, Koshihikari[25]. The results showed that, compared with the wild type 'Koshihikari', the introduced exogenous NiR gene made the plant grow better, while callus regeneration ability was also significantly improved.

Compared with the reference genome 'Dongzao', 'Linhuang No. 1' had an insert of 106 bases at the position 12517784 (CDS region) on chromosome NC_029681.1, whereas 'Muzao' had an insert of 104 bases at the same position; fragment insertion was verified by cloning. Insertion/deletion mutations are rarer than base substitutions in the evolution of organisms. If the sequence of the coding region has an indel mutation involving a base sequence which is not a multiple of three bases, it will cause serious consequences as a result of a frameshift mutation, which places the mutant under greater pressure in natural selection. In the present study, the domain of the protein encoded by the cloned NiR sequence was predicted, and it was found that the base insertion did not occur in the domain region. The expression levels of the LOC107427052 genes of 'Linhuang No. 1' and 'Muzao' were consistent with the changes measured in nitrite reductase activity during fruit development. However, the LOC107427052 gene expression level of 'Muzao' was significantly higher than that of 'Linhuang No. 1' during fruit development. The nitrite reductase activities and the nitrite content of 'Muzao' were significantly higher than those of 'Linhuang No. 1' at young fruit stage. But there was no significant difference in the product ammonia of nitrite reductase between the two varieties.

\section{Conclusions}

This present study was the first to explore the differences between different jujube varieties (Linhuang No. 1 and Muzao) by combining genome resequencing and transcriptomics. It found, by re-sequencing, that there are 664,129 mutation sites between 'Linhuang No. 1' and 'Muzao'. The characteristic mutation sites between 'Linhuang No. 1', 'Muzao' and 'Dongzao' were analyzed by principal component analysis. The genetic relationship between 'Linhuang No. 1' and 'Muzao' was closer than that with 'Dongzao'. A total of 431 differentially expressed genes was screened by transcriptomics, and 19 differentially expressed genes were screened by combining the transcriptomics with resequencing analysis. LOC107427052 (encoding nitrite reductase) was determined by KEGG enrichment analysis for further study. There was a large base insertion in the CDS region of the LOC107427052 gene, but the insertion was not in the domain region, as verified by the finding that the base insertion did not affect protein translation. Our study has laid a foundation for the analysis of genetic information and the comparative nitrite metabolism of 'Linhuang No. 1' and 'Muzao'.

\section{Materials And Methods Plant materials}

Fruits and leaves of 'Linhuang No. 1' and 'Muzao' were harvested from trees from the Jujube Germplasm Resource Nursery, Pomology Institute, Shanxi Agricultural University, Shanxi, China. The trees were 50 years old and cultivated according to current regulations for integrated fruit production. Fruits and leaves without disease or physical injuries were selected for sampling. All fruits and leaves were packed and delivered immediately to the laboratory at Shanxi Agricultural University.

\section{Genome re-sequencing}

Young leaves of 'Linhuang No. 1 ' and 'Muzao' were collected and stored at $-80^{\circ} \mathrm{C}$ prior to DNA isolation. Genomic DNA was extracted using a modified CTAB method. The sequencing library was prepared according to the standard protocol of Illumina and sequencing was conducted by Lianchuan bio, Beijing, China, using a Illumina NovaSeq 6000. Clean data were aligned to the jujube reference genome (https://ftp.ncbi.nlm.nih.gov/genomes/all/GCF/000/826/755/GCF_000826755.1_ZizJuj_1.1/GCF_000826755.1_ZizJuj_1.1_genomic.fna.gz), using Burrows-Wheeler Aligner (BWA) software[26]. Mark Duplicates in Picard (https://sourceforge.net/projects/picard/) was used to eliminate the PCR duplication. We used Genome Analysis Toolkit (GATK) for base re-calibration and re-alignment near insertion or deletion regions[26]. SAMtools was used to estimate reference genome coverage of SNP and indel variations for identification and analysis.

\section{Gene cloning and protein sequence alignment}


The target gene which encoding nitrite reductase (NiR) was amplified by cDNA from the pericarp of 'Linhuang No. 1' and 'Muzao' fruits. The primers used were forward primer ATGTCATCGTTCTCTGTTCGGTTT and reverse primer TCAAAACGGGTGTTTCCCTCGA. The amplified products were electrophoresed on $1 \%$ agarose gel and photographed with a gel imaging analysis system. The PCR products were recovered and purified according to the operation steps described in the Gel Recovery Kit (Takara, Japan). The recovered DNA fragment was ligated with a TA cloning vector and transformed into Escherichia coli DH5a. After 12-16 h inverted culture at $37^{\circ} \mathrm{C}$, single colonies were picked out and cultured in $2 \mathrm{ml}$ lysogeny broth (LB) liquid medium. After $5-6 \mathrm{~h}$ shake culture $(150 \mathrm{rpm})$ at $37^{\circ} \mathrm{C}, P s t l$ digestion was used for identification. The bacterial suspension was stored in a final concentration of about $25 \%(\mathrm{v} / \mathrm{v})$ glycerin, and a $100-\mu l$ aliquot of the bacterial suspension was sent to Sangon Biotech (Shanghai, China) for sequencing. The cloned sequences were subjected to domain analysis by TBtools[27] and NCBI-CD Search (https://www.ncbi.nlm.nih.gov/Structure/cdd/wrpsb.cgi).

\section{Quantitative real-time PCR validation of nitrite reductase gene expression level}

The quantitative real-time PCR (qPCR) was performed using the ChamQTM Universal SYBR qPCR Master Mix (Vazyme, Nanjing, China). The cDNA templates were reverse transcribed using total RNA extracted from the pericarp of fruit at different development periods from 'Linhuang No. 1' and 'Muzao'. Then nitrite reductase gene (LOC107427052) was PCR amplified. The primers were: Forward: GCAAATCCGTGGTGTGGTT and Reverse: CAGCAAGAGGGTTCCCAACT. The jujube Histon-H (HIS) gene (GenBank accession No. EU916201) was used as the internal reference for the gene expression analysis. The real-time PCR assay mix $(20 \mu \mathrm{L})$ consisted of $2 \mu \mathrm{L}$ cDNA sample (diluted 1:10), 10 $\mu \mathrm{L} 2 \times$ ChamQ Universal SYBR qPCR Master Mix (Vazyme, Nanjing, China), $0.6 \mu \mathrm{L}$ of each primer $(10 \mu \mathrm{M})$ and $6.8 \mu \mathrm{L}$ distilled deionized $\mathrm{H}_{2} \mathrm{O}$. Each PCR assay was run on an iCycler iQ Real-Time PCR Detection System (Bio-Rad, CA, USA), using the method described in previous study[28]. The $2^{-\triangle \triangle C q}$ method was used to calculate the relative amount of transcript template present in each PCR amplification mixture.

\section{Determination of the nitrite reductase (NiR) activity and concentrations of nitrite and ammonia nitrogen}

Determinations of the activity of nitrite reductase (NiR), and the concentrations of nitrite and ammonia nitrogen were carried out according to the Nitrite Reductase (NiR) Activity Assay Kit (BioBox, Beijing, China), Nitrite Content Assay Kit (BioBox, Beijing, China) and Plant Ammonia Nitrogen Content Assay Kit (BioBox, Beijing, China), respectively, following the manufacturer's instructions The pericarp of fruits harvested from 'Linhuang No. 1' and 'Muzao' at different stages of fruit development was taken as samples. Independent analysis of each cultivar $\times$ development stage combination was carried out three times.

\section{Data analysis}

SAS System 8.6 (SAS Institute, Cary, NC, USA) was used to analyze the data. Data analysis was carried out by one-way analysis of variance (ANOVA), with Student's $t$ test being used to make multiple pairwise comparisons to determine significant differences among samples. Differences with $p<0.05$ were considered to be statistically significant. Figures were constructed using Excel 2016, TBtools20 and Origin 9.0 (Microcal Software Inc., Northampton, MA, USA).

\section{Abbreviations}

SNP: single nucleotide polymorphisms; indel: insertion/deletion variants; SV: structural gene variants; CNV: copy number variants; DEGs: differentially expressed genes; KEGG: Kyoto Encyclopedia of Genes and Genomes; FPKM: Fragments per Kilobase Million; NiR: nitrite reductase; NR: nitrate reductase; CDS: coding sequence

\section{Declarations}

\section{Availability of data}

All data generated or analyzed during this study are included in the main paper and supplementary information files. In addition, Wholegenome re-sequencing and RNA-seq data in this study were uploaded to the National Center for Biotechnology Information (NCBI) Sequence Read Archive (SRA) database under accession number SRP307848 and SRP307646 respectively.

\section{Acknowledgements}

We thank BioMarker (Beijing, China) and Lianchuan (Shanghai, China) for help with the transcriptome sequencing and genome resequencing respectively.

Authors' contributions 
NL designed the experiment. NL, YS, JL, RH, XF and LL performed the experiments and analyzed the data. NL and LL wrote the manuscript. LL checked the manuscript. The author(s) read and approved the final manuscript.

\section{Funding}

This research was financially supported by the Shanxi Province Key Research and Development Projects (Grant number: 2020LYZY01) and Shanxi Agricultural University Science and technology innovation fund (Grant number: 2020BQ43 and 2020BQ28).

\section{Declarations}

Ethics approval and consent to participate

None.

\section{Consent for publication}

None.

\section{Competing interests}

The authors declare that they have no competing interests

\section{References}

1. Song J, Bi J, Chen Q, Wu X, Lyu Y, Meng X: Assessment of sugar content, fatty acids, free amino acids, and volatile profiles in jujube fruits at different ripening stages. Food chemistry 2019, 270:344-352.

2. Peng S: Current situation and Countermeasures of jujube production in China. In: The First National Symposium on Dry Fruit Production and Scientific Research Progress: 1998; Zanhuang, Heibei, China. 4.

3. Li X, Tong J, Wang H, Sun W: Ecological Adaptability of Ziziphus jujuba Mill. in North Shaanxi. Journal of Northwest Forestry University 2000, 15(02):13-18.

4. Guo Y, Shan G, Yang M: Regionalization culture of jujube tree in China. China Fruits 2002(04):46-48+53.

5. Lin J, Cheng Z, Xu M, Huang Z, Yang Z, Huang X, Zheng J, Lin T: Genome re-sequencing and bioinformatics analysis of a nutraceutical rice. Molecular genetics genomics 2015, 290:955-967.

6. Aliki X, Maria M, Christos B, Christos K, Maria FA, Evangelos K, Michail M, Chrysanthi P, Antonios Z, Konstantinos K et al: Whole genome re-sequencing of sweet cherry (Prunus avium L.) yields insights into genomic diversity of a fruit species. Horticulture Research 2020 , $7(1)$.

7. Yu Y, Fu J, Xu Y, Zhang J, Ren F, Zhao H, Tian S, Guo W, Tu X, Zhao J et al: Genome re-sequencing reveals the evolutionary history of peach fruit edibility. Nature communications 2018, 9(1).

8. Liu MJ, Zhao J, Cai QL, Liu GC, Wang JR, Zhao ZH, Liu P, Dai L, Yan G, Wang WJ: The complex jujube genome provides insights into fruit tree biology. Nature Communications 2014, 5:5315.

9. Huang J, Zhang C, Zhao X, Fei Z, Wan K, Zhang Z, Pang X, Yin X, Bai Y, Sun X et al: The Jujube Genome Provides Insights into Genome Evolution and the Domestication of Sweetness/Acidity Taste in Fruit Trees. PLOS Genetics 2016, 12(12).

10. Liu M, Wang J, Liu P, Zhao J, Zhao Z, Dai L, Li X, Liu Z: Historical Achievements and Frontier Advances in the Production and Research of Chinese Jujube (Ziziphus jujuba) in China. Acta Horticulturae Sinica 2015, 42(09):1683-1698.

11. Guo M, Zhang Z, Li S, Lian Q, Fu P, He Y, Qiao J, Xu K, Liu L, Wu M et al: Genomic analyses of diverse wild and cultivated accessions provide insights into the evolutionary history of jujube. Plant biotechnology journa/ 2020.

12. Zhang C, Huang J, Yin X, Lian C, Li X: Genetic diversity and population structure of sour jujube, Ziziphus acidojujuba. Tree Genetics \& Genomes 2015, 11(1).

13. Guo M, Zhang Z, Cheng Y, Li S, Shao P, Yu Q, Wang J, Xu G, Zhang X, Liu J et al: Comparative population genomics dissects the genetic basis of seven domestication traits in jujube. Horticulture research 2020, 7(1).

14. Sachidanandam R, Weissman D, Schmidt SC, Kakol JM, Stein LD: A map of human genome sequence variation containing 1.42 million single nucleotide polymorphisms. Nature: International weekly journal of science 2001, 409(6822).

15. Zhaolei Z, Mark G: Patterns of nucleotide substitution, insertion and deletion in the human genome inferred from pseudogenes. Nucleic Acids Research 2003, 31(18):5338. 
16. Wetterbom A, Sevov M, Cavelier L, Bergström TF: Comparative Genomic Analysis of Human and Chimpanzee Indicates a Key Role for Indels in Primate Evolution. Journal of Molecular Evolution 2006, 63(5).

17. Edwards JD, Lee VM, McCouch SR: Sources and predictors of resolvable indel polymorphism assessed using rice as a model Molecular Genetics and Genomics 2004, 271(3).

18. Britten RJ, Rowen L, Williams J, Cameron RA: Majority of Divergence between Closely Related DNA Samples Is Due to Indels. Proceedings of the National Academy of Sciences of the United States of America 2003, 100(8).

19. Long M, Betrán E, Thornton K, Wang W: The origin of new genes: glimpses from the young and old. Nature Reviews Genetics 2003, 4(1).

20. Jin J, Yang L, Fan D, Liu X, Hao Q: Comparative transcriptome analysis uncovers different heat stress responses in heat-resistant and heat-sensitive jujube cultivars. PLOS ONE 2020, 15(9):e0235763.

21. Zhang Q, Wang L, Liu Z, Zhao Z, Liu M: Transcriptome and metabolome profiling unveil the mechanisms of Ziziphus jujuba Mill. peel coloration. Food chemistry 2019, 312:125903.

22. Zhou H, He Y, Zhu Y, Li M, Song S, Bo W, Li Y, Pang X: Comparative transcriptome profiling reveals cold stress responsiveness in two contrasting Chinese jujube cultivars. BMC Plant Biology 2020, 20.

23. Silveira CM, Besson S, Moura I, Moura JJG, Almeida MG, Manessi-Zoupa E: Measuring the Cytochrome c Nitrite Reductase ActivityPractical Considerations on the Enzyme Assays. Bioinorganic Chemistry \& Applications 2010, 2010:1-8.

24. Sivasankar S, Rastogi R, Jackman L, Rothstein OS: Analysis of cis-acting DNA elements mediating induction and repression of the spinach nitrite reductase gene. Planta 1998, 206(1):66-71.

25. Ozawa K, Kawahigashi $\mathrm{H}$ : Positional cloning of the nitrite reductase gene associated with good growth and regeneration ability of calli and establishment of a new selection system for Agrobacterium -mediated transformation in rice ( Oryza sativa L.) Plant Science 2005, $170(2)$.

26. Li H, Handsaker B, Wysoker A, Fennell T, Ruan J, Homer N, Marth G, Abecasis G, Durbin R: The sequence alignment/map format and SAMtools. Bioinformatics 2009, 25(16):2078-2079.

27. Chen $\mathrm{C}$, Chen $\mathrm{H}$, Zhang $\mathrm{Y}$, Thomas HR, Frank MH, He Y, Xia R: TBtools: an integrative toolkit developed for interactive analyses of big biological data. Molecular plant 2020, 13(8):1194-1202.

28. Li N, Fu L, Song Y, Li J, Li L: Wax composition and concentration in jujube (Ziziphus jujuba Mill.) cultivars with differential resistance to fruit cracking. Journal of Plant Physiology 2020, 255:153294.

\section{Figures}




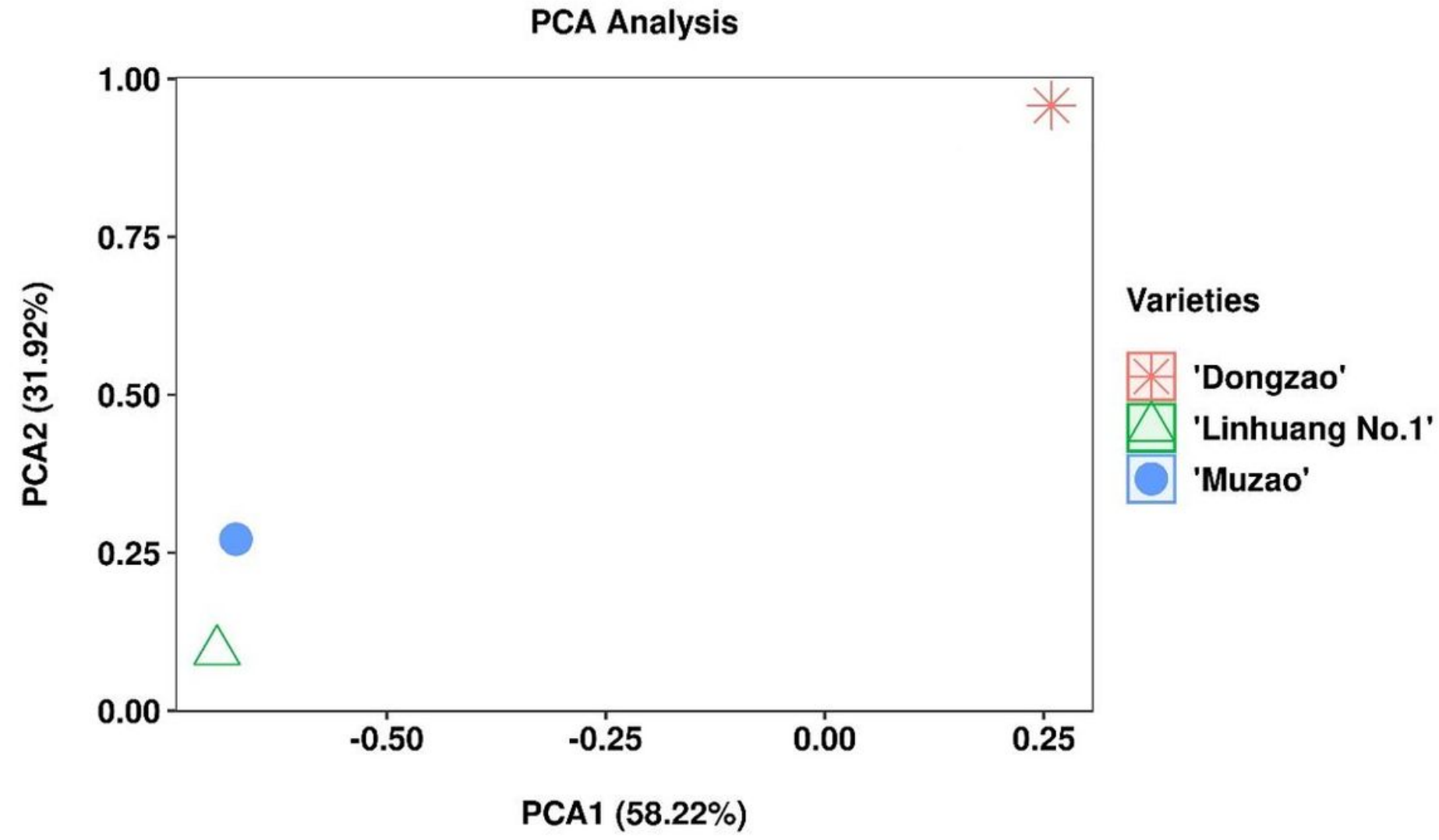

Figure 1

Principal component analysis (PCA) plot obtained from a similarity matrix among the Ziziphus jujuba Mill. accessions Linhuang No. 1 and Muzao, relative to the jujube genome reference cultivar Dongzao (ZizJuj_1.1, ID: 15586).
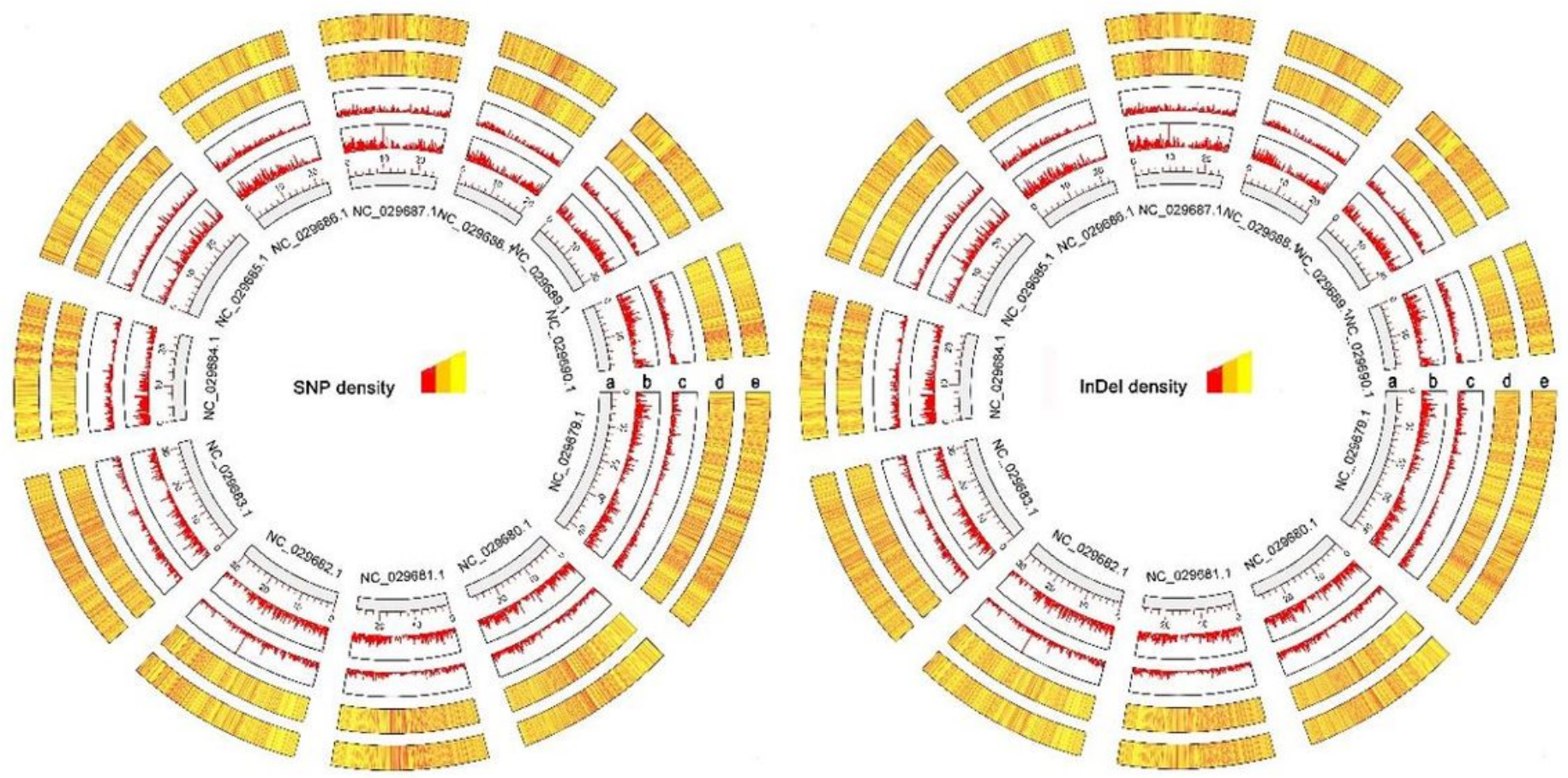

Figure 2 
SNP and indel density plots by chromosome for 'Linhuang No. 1' and 'Muzao' against the reference genome 'Dongzao' (a: Chromosome scale; b: Gene density of chromosome positive chain; c: Gene density of chromosome negative chain; d: 'Linhuang No.1'; e: 'Muzao')

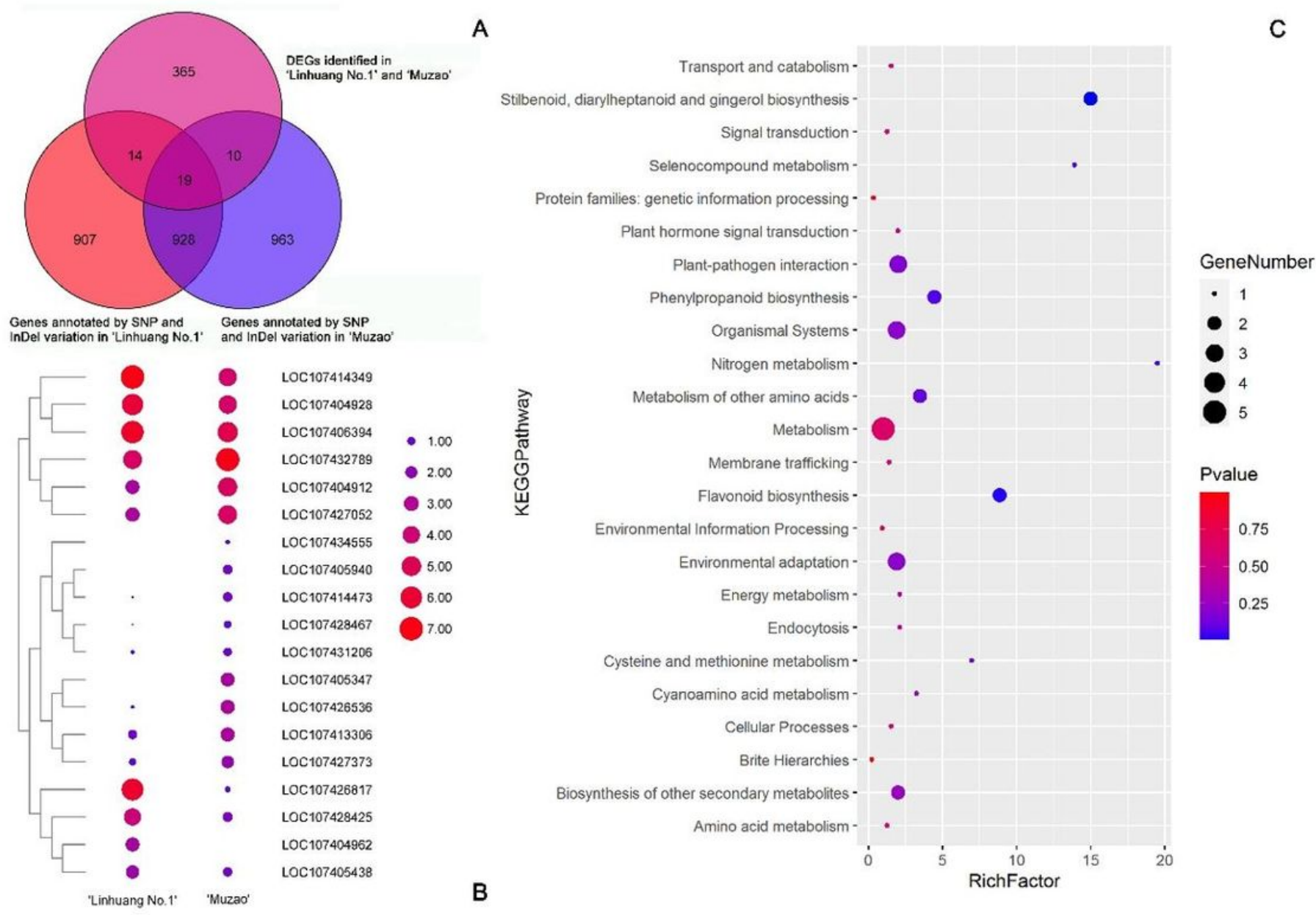

\section{Figure 3}

Venn diagram of 19 differentially expressed genes and SNP and indel annotated genes 


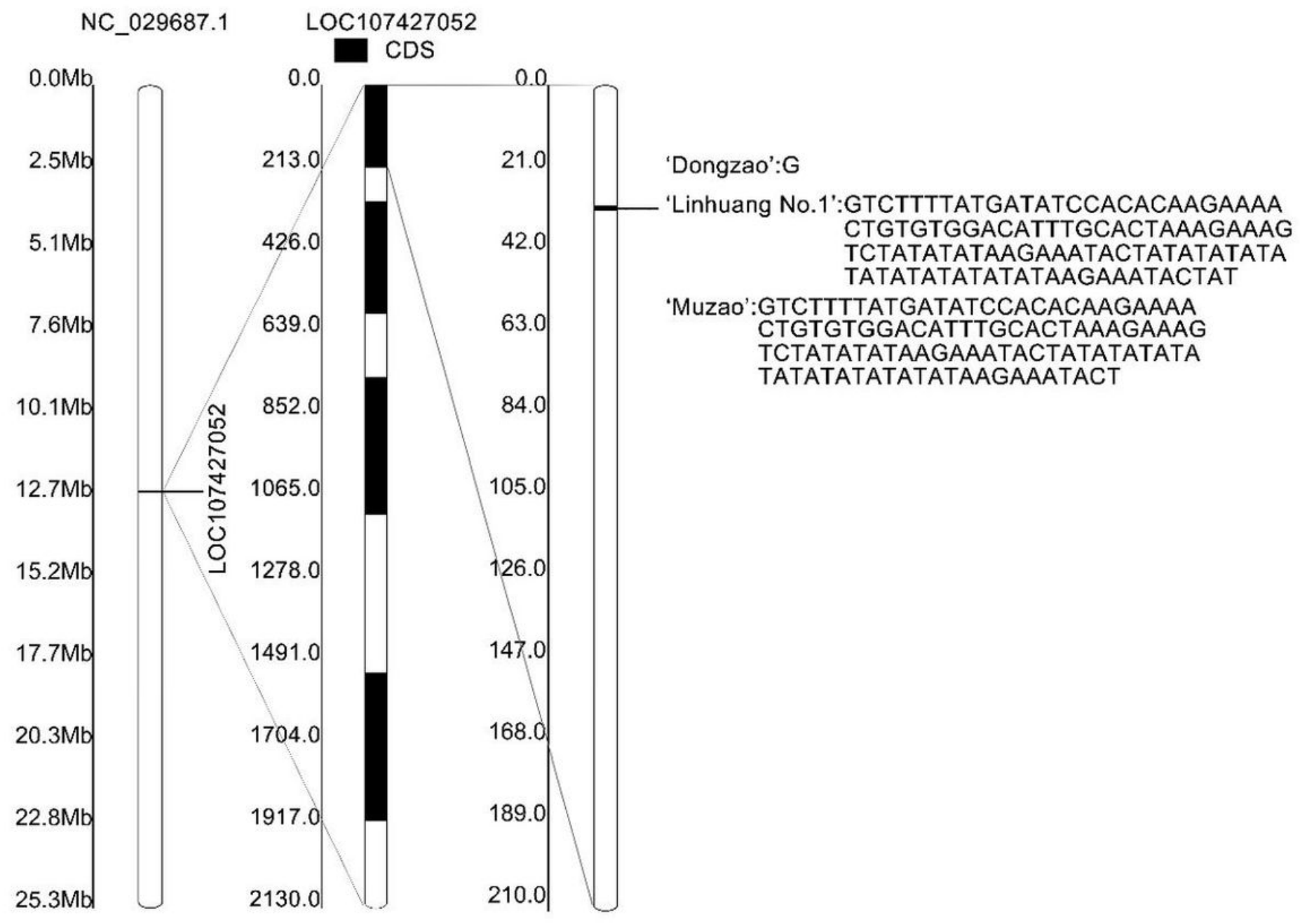

Figure 4

LOC107427052 mutation 


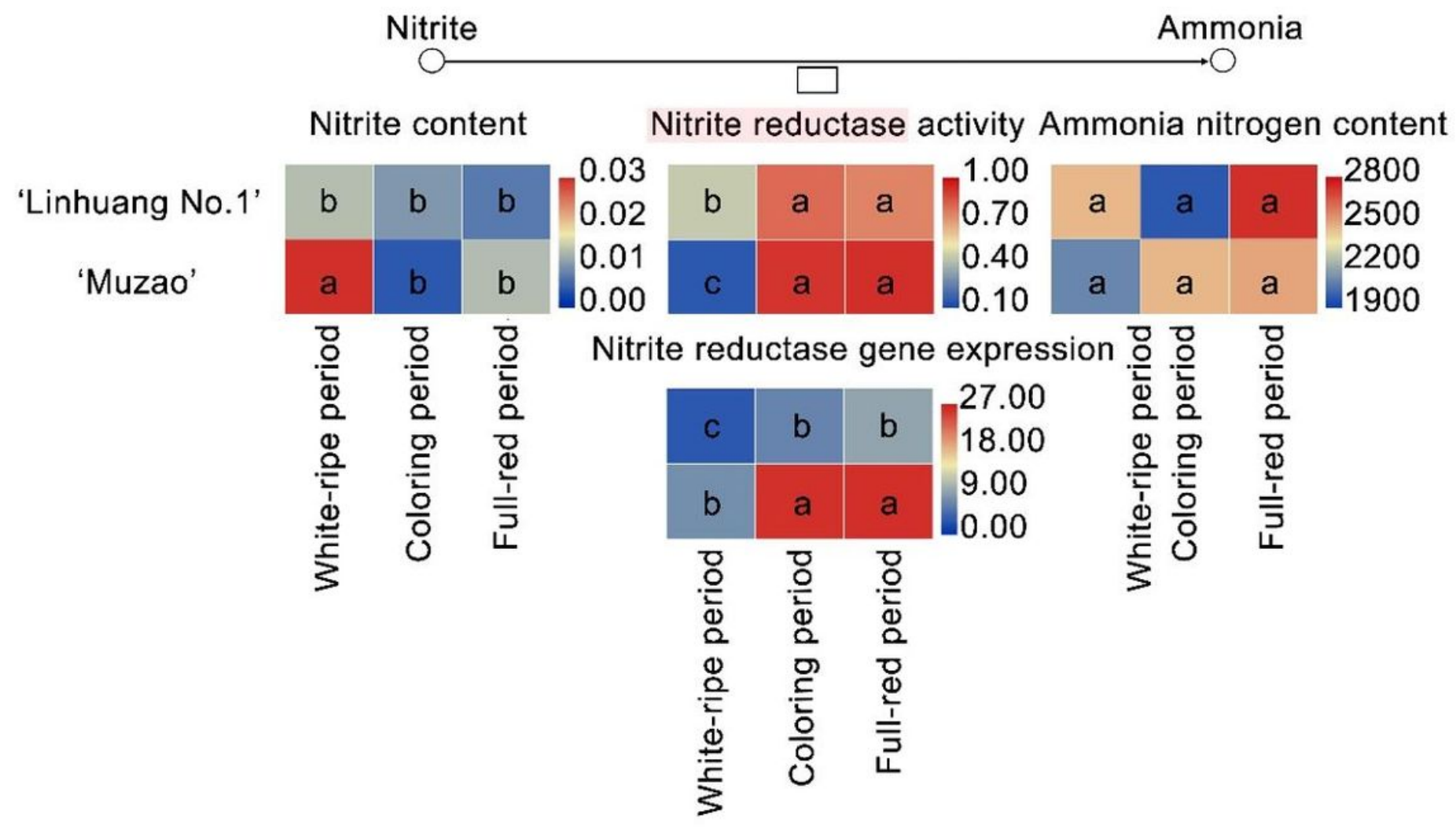

Figure 5

Levels of nitrite reductase (NiR) activity and gene expression, concentrations of nitrite and ammonia nitrogen at different stages of fruit development of the two cultivars. For a particular variable, any two samples with the same letter are not significantly different $(P>0.05)$

\section{Supplementary Files}

This is a list of supplementary files associated with this preprint. Click to download.

- Additionalfile1Tables1.xlsx

- Additionalfile2FigureS1.jpg

- Additionalfile3.txt

- Additionalfile4Figures2.jpg 